\title{
Knowledge and Perception of COVID-19 Pandemic during the First Wave (Feb-May 2020): A Cross-Sectional Study among Italian Healthcare Workers
}

\author{
Caterina Rizzo ${ }^{1}$, Ilaria Campagna ${ }^{2}$, Elisabetta Pandolfi ${ }^{2, *}$, Ileana Croci ${ }^{2}$, Luisa Russo ${ }^{2}$ D, Sara Ciampini ${ }^{3}$, \\ Francesco Gesualdo ${ }^{2}$, Alberto Eugenio Tozzi ${ }^{2}$ D, Lara Ricotta ${ }^{4}$, Massimiliano Raponi ${ }^{4}$ \\ and Marta Luisa Ciofi Degli Atti ${ }^{1}$
}

check for updates

Citation: Rizzo, C.; Campagna, I.;

Pandolfi, E.; Croci, I.; Russo, L.;

Ciampini, S.; Gesualdo, F.; Tozzi, A.E.;

Ricotta, L.; Raponi, M.; et al.

Knowledge and Perception of

COVID-19 Pandemic during the First

Wave (Feb-May 2020):

A Cross-Sectional Study among Italian Healthcare Workers. Int. J.

Environ. Res. Public Health 2021, 18,

3767. https://doi.org/10.3390/

ijerph18073767

Academic Editor: Riccardo Polosa

Received: 16 February 2021

Accepted: 1 April 2021

Published: 4 April 2021

Publisher's Note: MDPI stays neutral with regard to jurisdictional claims in published maps and institutional affiliations.

Copyright: (c) 2021 by the authors. Licensee MDPI, Basel, Switzerland. This article is an open access article distributed under the terms and conditions of the Creative Commons Attribution (CC BY) license (https:// creativecommons.org/licenses/by/ $4.0 /)$.
1 Clinical Pathways and Epidemiology, Bambino Gesù Children's Hospital IRCSS, Viale di Villa Pamphili 100 , 00152 Rome, Italy; caterina1.rizzo@opbg.net (C.R.); marta.ciofidegliatti@opbg.net (M.L.C.D.A.)

2 Multifactorial Disease and Complex Disease Research Area, Bambino Gesù Children's Hospital IRCSS, Viale di Villa Pamphili 100, 00152 Rome, Italy; ilaria.campagna@opbg.net (I.C.); ileana.croci@opbg.net (I.C.); luisa.russo@opbg.net (L.R.); francesco.gesualdo@opbg.net (F.G.); albertoeugenio.tozzi@opbg.net (A.E.T.)

3 Public Health Service, Local Health Authority Rome 1, Borgo Santo Spirito 3, 00193 Rome, Italy; drsara.ciampini@gmail.com

4 Medical Direction, Bambino Gesù Children's Hospital IRCCS, Piazza S. Onofrio 4, 00165 Rome, Italy; lara.ricotta@opbg.net (L.R.); massimiliano.raponi@opbg.net (M.R.)

* Correspondence: elisabetta.pandolfi@opbg.net

Abstract: Italy was the first country in Europe to face the coronavirus pandemic. The aim of the study was to analyze healthcare workers' (HCWs) level of information, practice, and risk perception towards COVID-19. We set up a cross-sectional study through SurveyMonkey ${ }^{\circledR}$ and distributed the link through Facebook and Whatsapp closed groups. The research instrument was a 31 items questionnaire distributed using Facebook and Whatsapp. It was conducted in Italy from February to May 2020. The study participants were general practitioners, pediatricians and other health professionals. A total of 958 participants were included: 320 (33.4\%) general practitioners, 248 (25.9\%) pediatricians and $390(40.7 \%)$ other health professionals. The highest response rate was from Northern Italy (48.1\%), followed by Central Italy (29.9\%) and Southern Italy $(22.0 \%)$. Less than a half (46\%) of respondents felt they had a good level of information of COVID-19 case definition and of national prevention guidelines. Respondents reported to have changed their clinical practice; particularly, they increased the use of masks $(87.1 \%, p<0.001)$, disinfection and sanitization of doctors' offices $(75.8 \%, p<0.001)$, the use of protective glasses $(71.2 \%, p<0.001)$, alcoholic hand solution $(71.2 \%$, $p<0.001)$, and hand washing $(31.8 \%, p=0.028)$. HCWs are at high risk of infection; less than a half of them felt adequately prepared to face COVID-19 pandemic, so they need extensive information and awareness of the disease to take adequate precautionary measures, and they are crucial to disseminate good practices.

Keywords: information; attitude; COVID-19; SARS-CoV-2; healthcare workers

\section{Background}

On 31 December, 2019, the World Health Organization (WHO) received reports from the Chinese health authorities about the presence of pneumonia cases of unknown cause detected in the city of Wuhan, in the Chinese province of Hubei [1]. Subsequently, the Chinese health authorities identified a new coronavirus, Severe Acute Respiratory Syndrome-Coronavirus 2 (SARS-CoV-2), as responsible for the coronavirus disease 2019 (COVID-19) [2].

The first two cases of the COVID-19 pandemic in Italy, which tested positive for the SARS-CoV-2 virus in Rome, were confirmed on 30th January, both with a travel history to Wuhan, China. On 21 February, 2020, the Italian National Institute of Health confirmed the 
first autochthonous case in Northern Italy (Codogno city-Lombardy region) in a critically ill, hospitalized young man with no travel history to known areas of viral circulation or links to a probable or confirmed COVID-19 case [3-5].

This unexpected finding unveiled ongoing transmission in several municipalities in the Lombardy Region [6]. In subsequent days and weeks, case counts and death tolls increased rapidly, first in Northern Italy, and then in the rest of the country. The Italian government imposed increasingly strict physical distancing measures, starting with the closure of 10 municipalities in the Lodi Province (Lombardy) and one in the Padua Province (Veneto) on the 22nd of February 2020 [5-7]. This culminated in a national lockdown declared on 10 March 2020 and ended on 3 May 2020. COVID-19 is spread by human-tohuman transmission through droplets and direct contact; it has an incubation period of 2-14 days [8-13].

The clinical presentation of the Sars-CoV-2 infection varies from asymptomatic to very severe pneumonia with acute respiratory distress syndrome, septic shock and multi-organ failure, which can cause death [14-16]. To date, applying preventive measures to control COVID-19 infection is the most critical intervention.

Healthcare workers (HCWs) are at high risk of infection and they may also contribute to the spread of the disease. Infection prevention and control (IPC) during health care practices when COVID-19 is suspected or confirmed is crucial in order to protect HCWs and fragile patients [17]. The rate of infection reported in HCWs varies across countries [18-21]. In China, HCWs accounted for $3.8 \%$ of all cases, with $14.8 \%$ of these having severe/critical disease despite their young age and few comorbidities $[22,23]$. Other studies, however, reported lower illness severity in HCWs and identified PPE use as the main factor associated with decreased infection risk [24].

Measuring scope of information, attitudes and risk perception in regards to IPC could help to predict HCWs' behaviors in applying preventive and control measures.

Even though the Italian Ministry of Health published guidelines and developed strong initiatives for IPC in healthcare facilities to prevent the spread of the disease, it is crucial to understand if those guidelines were really applied, particularly among HCWs [25].

The aim of the study was to the measure information level of healthcare workers, their risk perception towards the pandemic and their practice (behavior change) in applying COVID-19 nonpharmaceutical preventive measures. With this purpose, we conducted a cross-sectional study at the very beginning of the autochthonous circulation of SARS-CoV-2 in Italy.

\section{Methods}

\subsection{Study Design}

This cross-sectional study was conducted in Italy from the end of February to the first week of May 2020, and it was coordinated by Bambino Gesù Children's Hospital in Rome.

\subsection{Data Collection Procedures}

The survey was set up using Survey Monkey ${ }^{\circledR}$ and the link to the survey was circulated online through Facebook and WhatsApp closed groups. The study participants were HCWs, including general practitioners, pediatricians, consultants, postgraduate trainees, and other health professionals (nurses, midwives, physiotherapists, etc.).

Data was collected using a structured questionnaire that comprised 31 predefined responses, including the demographic, scope of information, awareness and practice assessment sections. The developed questionnaire was tested among different HCWs in the Bambino Gesù Children's Hospital, and open-ended questions were limited to reduce information bias.

The Section 1 of the questionnaire consisted of six questions regarding demographic details and professional profile. Section 2 consisted of two questions about the risk perception of HCWs and patients. Section 3 consisted of seven questions focusing on the 
information level of HCWs. Section 4 had 15 questions regarding attitudes and practices, precautions and procedures to contain the virus.

\subsection{Sample Size}

The sample size for the survey was calculated according to the formula adopted in the Raosoft software (http:/ / www.raosoft.com/samplesize.html, accessed on 12 November 2020). Setting the expected proportion of the outcome found in each question of the study at $50 \%$ with an accepted margin of error of $5 \%$, we obtained a total sample of 377 individuals, with a confidence level of $95 \%$.

\subsection{Definitions}

We considered two main outcomes in the analysis: information and behavior change. Information was defined as at least an affirmative response to one of the following four questions:

- Do you believe that information released by international health authorities regarding the pandemic from COVID-19 in China has been clear enough?

- Do you believe that information disseminated by national and regional health authorities regarding the risks associated with COVID-19 for the Italian population has been sufficiently clear?

- Do you think that the definition of a suspected case of COVID-19 infection is sufficiently clear?

- Do you think you have been sufficiently informed by the national health authorities on how to behave if you are faced with a suspected case of COVID-19?

Practice (behavior change) was defined as at least an affirmative response to one of the following three questions:

- $\quad$ Since the start of the COVID-19 pandemic, have you changed the way you work?

- $\quad$ Since the start of the COVID-19 pandemic, has there been any impact in the organization of visits?

- $\quad$ Since the beginning of the COVID-19 pandemic, has there been any impact in your relationship with patients?

Questions on the risk perception for being in contact with COVID-19 for HCWs and their patients were measured using the Likert scale ranging from 0 to 10 (no risk and high risk, respectively); the answers were then categorized into five groups according to the percentile distribution to better show the results in the graph.

For the two questions based on the Likert scale, the data were divided in five groups according to a $20 \%$ difference.

The first question ("Are the patients you come in contact with, scared of the COVID-19 pandemic?") was categorized as follows: group $1\left(0-20^{\circ}\right)$ was from $0-5$ points of the Likert scale; group $2\left(21^{\circ}-40^{\circ}\right)$ was from $6-7$ points of the Likert scale; group $3\left(41^{\circ}-60^{\circ}\right)$ corresponded to 8 points of the Likert scale; group $4\left(61^{\circ}-80^{\circ}\right)$ corresponded to 9 points of the Likert scale; group $5\left(81^{\circ}-100^{\circ}\right)$ corresponded to 10 points of the Likert scale.

The second question ("Based on your views, what is the risk of visiting a patient with SARS-CoV-2 in the coming weeks?") was categorized as follows: group $1\left(0-20^{\circ}\right)$ was from $0-5$ points of the Likert scale; group $2\left(21^{\circ}-40^{\circ}\right)$ corresponded to 6 points of the Likert scale; group $3\left(41^{\circ}-60^{\circ}\right)$ was from $7-8$ points of the Likert scale; group $4\left(61^{\circ}-80^{\circ}\right)$ corresponded to 9 points of the Likert scale; group $5\left(81^{\circ}-100^{\circ}\right)$ corresponded to 10 points of the Likert scale.

We considered the pre-lockdown period from 26 February to 10 March 2020 and the lockdown period from 12 March to 3 May 2020 [26].

\subsection{Statistical Analysis}

Univariate differences were tested using Pearson's Chi square test for categorical variables and nonparametric Wilcoxon Mann-Whitney test for independent continuous 
variables. We carried out multivariate ordered logistic regression to investigate the association between the socioeconomic characteristics, the variables investigated in the questionnaire and two outcomes (information and behavior change).

We carried out multiple imputations with chained equations [27] to generate values for missing data points such as sex, age, region and attitude to face the pandemic. The percentage of missing data was $25 \%$.

All variables included in the models as predictors of outcomes were used to predict missing values [27,28]. Data were assumed to be "missing at random" [28]. Twenty-five datasets were imputed. Outcomes were not imputed. Data analysis was performed with STATA 13.0 SE (Stata Corporation, College Station, TX, USA).

\section{Results}

A total of 958 participants were included. Of these, 320 (33.4\%) were general practitioners, $248(25.9 \%)$ were pediatricians and $390(40.7 \%)$ were other health professionals. Most responders filled in the questionnaire during the pre-lockdown period $(72.4 \%)$. The characteristics of the participants are shown in Table 1. Most respondents were from Northern Italy (48.1\%), followed by Central Italy (29.9\%) and, lastly, Southern Italy (22.0\%), and the majority of respondents were female (61.8\%). Most of HCWs work in an urban environment (62\%) (Figure 1).

Patients were reportedly more afraid of COVID-19 during the lockdown period $(15.8 \%)$ than in the pre-lockdown period $(8.4 \%)(p<0.001)$ (Table 2). Health professionals reported a higher level of perceived risk of contracting COVID-19 from their patients than pediatricians (Figure 2).

According to respondents, the risk of having contact with a patient affected by COVID19 was far higher in the lockdown period than the pre-lockdown period (19.4\% vs $10.2 \%$, $p=0.002$ ) (Table 2). This risk was higher for general practitioners than for pediatricians and other health professionals, but this difference was not statistically significant (Figure 3).

Table 1. Participants' demographics $(n=958)$.

\begin{tabular}{|c|c|c|c|c|c|c|c|}
\hline & \multicolumn{2}{|c|}{$\begin{array}{c}\text { Total } \\
(n=958)\end{array}$} & \multicolumn{2}{|c|}{$\begin{array}{l}\text { Pre-Lockdown } \\
\quad(n=694)\end{array}$} & \multicolumn{2}{|c|}{$\begin{array}{l}\text { Lockdown } \\
(n=264)\end{array}$} & \multirow[b]{2}{*}{$p$} \\
\hline & $\mathbf{n}$ & $\%$ & $\mathbf{n}$ & $\%$ & $\mathbf{n}$ & $\%$ & \\
\hline Type of participants & & & & & & & 0.022 \\
\hline Non medical staff & 390 & 40.7 & 267 & 38.5 & 123 & 46.6 & \\
\hline Medical staff & 568 & 59.3 & 427 & 61.5 & 141 & 53.4 & \\
\hline Profession & & & & & & & $<0.001$ \\
\hline Allied healthcare & 390 & 40.7 & 267 & 38.5 & 123 & 46.6 & \\
\hline General Practitioner & 320 & 33.4 & 216 & 31.1 & 104 & 39.4 & \\
\hline Pediatrician & 248 & 25.9 & 211 & 30.4 & 37 & 14.0 & \\
\hline Age (range) & & & & & & & 0.043 \\
\hline Median (IQR) & & & & & & & \\
\hline Age (in years) & & & & & & & 0.041 \\
\hline$\leq 35$ & 215 & 24.1 & 170 & 26.2 & 45 & 18.7 & \\
\hline $36-45$ & 150 & 16.8 & 111 & 17.1 & 39 & 16.2 & \\
\hline $46-55$ & 136 & 15.3 & 87 & 13.4 & 49 & 20.3 & \\
\hline $56-65$ & 312 & 35.0 & 227 & 34.9 & 85 & 35.3 & \\
\hline$\geq 66$ & 78 & 8.8 & 55 & 8.5 & 23 & 9.5 & \\
\hline Sex & & & & & & & $<0.001$ \\
\hline Male & 341 & 38.2 & 221 & 34.0 & 120 & 49.6 & \\
\hline Female & 551 & 61.8 & 429 & 66.0 & 122 & 50.4 & \\
\hline
\end{tabular}


Table 1. Cont

\begin{tabular}{|c|c|c|c|c|c|c|c|}
\hline & \multicolumn{2}{|c|}{$\begin{array}{c}\text { Total } \\
(n=958)\end{array}$} & \multicolumn{2}{|c|}{$\begin{array}{l}\text { Pre-Lockdown } \\
\quad(n=694)\end{array}$} & \multicolumn{2}{|c|}{$\begin{array}{l}\text { Lockdown } \\
(n=264)\end{array}$} & \multirow[b]{2}{*}{$p$} \\
\hline & $\mathbf{n}$ & $\%$ & $\mathbf{n}$ & $\%$ & n & $\%$ & \\
\hline Area & & & & & & & 0.545 \\
\hline North Italy & 427 & 48.1 & 318 & 48.9 & 109 & 45.8 & \\
\hline Central Italy & 266 & 29.9 & 195 & 30.0 & 71 & 29.8 & \\
\hline South Italy & 195 & 22.0 & 137 & 21.1 & 58 & 24.4 & \\
\hline Organization * & & & & & & & 0.828 \\
\hline Hospital & 222 & 57.2 & 155 & 58.1 & 67 & 55.4 & \\
\hline Residential Care Facility & 60 & 15.5 & 39 & 14.6 & 21 & 17.4 & \\
\hline Private institution & 36 & 9.3 & 27 & 10.1 & 9 & 7.4 & \\
\hline Community healthcare center & 33 & 8.5 & 22 & 8.2 & 11 & 9.1 & \\
\hline Other & 37 & 9.5 & 24 & 9.0 & 13 & 10.7 & \\
\hline
\end{tabular}

Table 2. Information level, risk perception and practice of HCWs by survey study period in Italy, univariate analysis.

\begin{tabular}{|c|c|c|c|c|c|c|c|}
\hline & \multicolumn{2}{|c|}{$\begin{array}{c}\text { Total } \\
(n=958)\end{array}$} & \multicolumn{2}{|c|}{$\begin{array}{l}\text { Pre-Lockdown } \\
\quad(n=694)\end{array}$} & \multicolumn{2}{|c|}{$\begin{array}{l}\text { Lockdown } \\
(n=264)\end{array}$} & \multirow[b]{2}{*}{$p$} \\
\hline & $\mathbf{n}$ & $\%$ & $\mathrm{n}$ & $\%$ & $\mathbf{n}$ & $\%$ & \\
\hline $\begin{array}{l}\text { Are the patients you come in contact with, } \\
\text { scared of the COVID-19 pandemic? }\end{array}$ & & & & & & & $<0.001$ \\
\hline Not at all frightened & 237 & 25.2 & 197 & 28.9 & 40 & 15.4 & \\
\hline A little scared & 274 & 29.1 & 219 & 32.1 & 55 & 21.2 & \\
\hline Neutral & 221 & 23.5 & 149 & 21.8 & 72 & 27.8 & \\
\hline Quite frightened & 111 & 11.8 & 60 & 8.8 & 51 & 19.7 & \\
\hline Very scared & 98 & 10.4 & 57 & 8.4 & 41 & 15.8 & \\
\hline $\begin{array}{l}\text { In the last week, which was for you the risk } \\
\text { to come in contact with patients affected } \\
\text { by COVID-19? }\end{array}$ & & & & & & & 0.002 \\
\hline Absence of risk & 264 & 31.1 & 216 & 33.5 & 48 & 23.3 & \\
\hline Minimal risk & 87 & 10.2 & 69 & 10.7 & 18 & 8.7 & \\
\hline Neutral & 290 & 34.1 & 219 & 34.0 & 71 & 34.5 & \\
\hline Moderate risk & 103 & 12.1 & 74 & 11.5 & 29 & 14.1 & \\
\hline High risk & 106 & 12.5 & 66 & 10.2 & 40 & 19.4 & \\
\hline \multicolumn{8}{|l|}{$\begin{array}{l}\text { What containment measures were put in } \\
\text { place in your work place? }\end{array}$} \\
\hline Isolation of the patient (yes) & 72 & 85.7 & 29 & 96.7 & 43 & 82.7 & 0.063 \\
\hline Contact quarantine (yes) & 40 & 47.6 & 18 & 60.0 & 22 & 40.7 & 0.090 \\
\hline $\begin{array}{l}\text { Administration of nasal swabs for } \\
\text { close contacts }\end{array}$ & 35 & 41.7 & 15 & 50.0 & 20 & 35.2 & 0.185 \\
\hline Healthcare worker quarantine (yes) & 25 & 29.8 & 12 & 40.0 & 13 & 24.1 & 0.126 \\
\hline Social containment measures (yes) & 21 & 25.0 & - & - & 21 & 25.0 & $<0.001$ \\
\hline Use of PPE (yes) & 4 & 4.8 & 2 & 6.7 & 2 & 3.7 & 0.143 \\
\hline
\end{tabular}


Table 2. Cont.

\begin{tabular}{|c|c|c|c|c|c|c|c|}
\hline & \multicolumn{2}{|c|}{$\begin{array}{c}\text { Total } \\
(n=958)\end{array}$} & \multicolumn{2}{|c|}{$\begin{array}{l}\text { Pre-Lockdown } \\
\qquad(n=694)\end{array}$} & \multicolumn{2}{|c|}{$\begin{array}{l}\text { Lockdown } \\
(n=264)\end{array}$} & \multirow[b]{2}{*}{$p$} \\
\hline & $\mathbf{n}$ & $\%$ & $\mathbf{n}$ & $\%$ & $\mathbf{n}$ & $\%$ & \\
\hline $\begin{array}{l}\text { Since the start of the COVID-19 pandemic, } \\
\text { have you changed the way you work? }\end{array}$ & & & & & & & $<0.001$ \\
\hline Yes, absolutely & 519 & 55.8 & 313 & 46.3 & 206 & 81.1 & \\
\hline Yes, moderately & 335 & 36.0 & 291 & 43.1 & 44 & 17.3 & \\
\hline No, not really & 51 & 5.5 & 49 & 7.2 & 2 & 0.8 & \\
\hline No, not at all & 21 & 2.3 & 19 & 2.8 & 2 & 0.8 & \\
\hline I don't know & 4 & 0.4 & 4 & 0.6 & - & - & \\
\hline \multicolumn{8}{|l|}{$\begin{array}{c}\text { What have you changed in your } \\
\text { clinical practice? }{ }^{*}\end{array}$} \\
\hline Increased frequency of handwashing (yes) & 256 & 26.7 & 172 & 24.8 & 84 & 31.8 & 0.028 \\
\hline Increased office disinfection (yes) & 624 & 65.1 & 424 & 61.1 & 200 & 75.8 & $<0.001$ \\
\hline Increased use of masks (yes) & 628 & 65.6 & 398 & 57.4 & 230 & 87.1 & $<0.001$ \\
\hline Increased use of protective glasses (yes) & 440 & 45.9 & 252 & 36.3 & 188 & 71.2 & $<0.001$ \\
\hline $\begin{array}{l}\text { Increased use of alcohol based hand } \\
\text { solution (yes) }\end{array}$ & 467 & 48.8 & 279 & 40.2 & 188 & 71.2 & $<0.001$ \\
\hline $\begin{array}{l}\text { Do you believe that the information } \\
\text { released by international health authorities } \\
\text { regarding the COVID-19 pandemic in } \\
\text { China has been clear enough? }\end{array}$ & & & & & & & 0.100 \\
\hline Yes, absolutely & 152 & 18.0 & 111 & 18.2 & 41 & 17.7 & \\
\hline Yes, moderately & 330 & 39.2 & 255 & 41.7 & 75 & 32.3 & \\
\hline No, not really & 283 & 33.6 & 191 & 31.3 & 92 & 39.7 & \\
\hline No, not at all & 71 & 8.4 & 49 & 8.0 & 22 & 9.5 & \\
\hline I don't know & 7 & 0.8 & 5 & 0.8 & 2 & 0.9 & \\
\hline $\begin{array}{l}\text { Do you believe that the information } \\
\text { disseminated by national and regional } \\
\text { health authorities regarding the risks } \\
\text { associated with COVID-19 for the Italian } \\
\text { population have been sufficiently clear? }\end{array}$ & & & & & & & 0.129 \\
\hline Yes, absolutely & 174 & 20.9 & 122 & 20.1 & 52 & 23.2 & \\
\hline Yes, moderately & 354 & 42.5 & 271 & 44.6 & 83 & 37.1 & \\
\hline No, not really & 222 & 26.7 & 163 & 26.8 & 59 & 26.3 & \\
\hline No, not at all & 78 & 9.4 & 49 & 8.1 & 29 & 12.9 & \\
\hline I don't know & 4 & 0.5 & 3 & 0.5 & 1 & 0.5 & \\
\hline $\begin{array}{l}\text { Do you think that the definition of a } \\
\text { suspected case of a COVID-19 infection is } \\
\text { sufficiently clear? }\end{array}$ & & & & & & & 0.750 \\
\hline Yes, absolutely & 185 & 22.4 & 133 & 22.1 & 52 & 23.4 & \\
\hline Yes, moderately & 353 & 42.8 & 265 & 43.9 & 88 & 39.6 & \\
\hline No, not really & 235 & 28.5 & 170 & 28.2 & 65 & 29.3 & \\
\hline No, not at all & 48 & 5.8 & 32 & 5.3 & 16 & 7.2 & \\
\hline I don't know & 4 & 0.5 & 3 & 0.5 & 1 & 0.5 & \\
\hline
\end{tabular}


Table 2. Cont.

\begin{tabular}{|c|c|c|c|c|c|c|c|}
\hline & \multicolumn{2}{|c|}{$\begin{array}{c}\text { Total } \\
(n=958)\end{array}$} & \multicolumn{2}{|c|}{$\begin{array}{l}\text { Pre-Lockdown } \\
\quad(n=694)\end{array}$} & \multicolumn{2}{|c|}{$\begin{array}{l}\text { Lockdown } \\
(n=264)\end{array}$} & \multirow[b]{2}{*}{$p$} \\
\hline & $\mathbf{n}$ & $\%$ & $\mathbf{n}$ & $\%$ & $\mathbf{n}$ & $\%$ & \\
\hline $\begin{array}{l}\text { Do you think you have been sufficiently } \\
\text { informed by the national health authorities } \\
\text { on how to behave if you are faced with a } \\
\text { suspected COVID-19 case? }\end{array}$ & & & & & & & 0.477 \\
\hline Yes, absolutely & 195 & 23.3 & 147 & 24.3 & 48 & 20.9 & \\
\hline Yes, moderately & 375 & 44.9 & 276 & 45.5 & 99 & 43.0 & \\
\hline No, not really & 204 & 24.4 & 138 & 22.8 & 66 & 28.7 & \\
\hline No, not at all & 59 & 7.1 & 43 & 7.1 & 16 & 7.0 & \\
\hline I don't know & 3 & 0.4 & 2 & 0.3 & 1 & 0.4 & \\
\hline
\end{tabular}

Which of the following protective devices

is most suitable to avoid the risk of

$<0.001$

transmission of COVID-19?

\begin{tabular}{|c|c|c|c|c|c|c|c|}
\hline Surgical mask & 63 & 7.5 & 24 & 3.9 & 39 & 16.8 & \\
\hline FFP1 mask & 17 & 2.0 & 13 & 2.1 & 4 & 1.7 & \\
\hline FFP2 mask & 16 & 1.9 & 7 & 1.1 & 9 & 3.9 & \\
\hline FFP3 mask & 642 & 75.9 & 510 & 83.1 & 132 & 56.9 & \\
\hline Gas-masks & 1 & 0.1 & 1 & 0.2 & - & - & \\
\hline All the above & 107 & 12.7 & 59 & 9.6 & 48 & 20.7 & \\
\hline \multicolumn{8}{|l|}{$\begin{array}{l}\text { How do you keep yourself informed about } \\
\text { operational guidelines? }\end{array}$} \\
\hline Emails sent by health authorities (yes) & 595 & 62.1 & 450 & 64.8 & 145 & 54.9 & 0.005 \\
\hline $\begin{array}{l}\text { Proactive search for information on official } \\
\text { institutions' websites (yes) }\end{array}$ & 521 & 54.4 & 360 & 51.9 & 161 & 61.0 & 0.011 \\
\hline $\begin{array}{l}\text { Proactive search for information through } \\
\text { Ministry of Health toll-free number (yes) }\end{array}$ & 156 & 16.3 & 113 & 16.3 & 43 & 16.3 & 0.998 \\
\hline $\begin{array}{l}\text { Emails received from scientific } \\
\text { companies (yes) }\end{array}$ & 262 & 27.4 & 190 & 27.4 & 72 & 27.3 & 0.974 \\
\hline $\begin{array}{l}\text { Exchange of information with other } \\
\text { colleagues (yes) }\end{array}$ & 496 & 51.8 & 339 & 48.8 & 157 & 59.9 & 0.003 \\
\hline Medical-scientific publications (yes) & 291 & 30.4 & 203 & 29.2 & 88 & 33.3 & 0.220 \\
\hline Social networks (yes) & 151 & 15.8 & 116 & 16.7 & 35 & 13.3 & 0.189 \\
\hline From patients (yes) & 7 & 0.7 & 4 & 0.6 & 3 & 1.1 & 0.363 \\
\hline
\end{tabular}

Which of the following social networks do you find most reliable to follow updates on the COVID-19 pandemic?

\begin{tabular}{ccccccccc}
\hline Facebook (yes) & 111 & 13.2 & 89 & 14.6 & 22 & 9.6 & 0.055 \\
\hline LinkedIn (yes) & 22 & 2.6 & 10 & 1.6 & 12 & 5.2 & 0.004 \\
\hline Instagram (yes) & 16 & 1.9 & 12 & 2.0 & 4 & 1.7 & 0.829 \\
\hline Twitter (yes) & 28 & 3.3 & 18 & 2.9 & 10 & 4.3 & 0.315 \\
\hline They are not reliable for updates (yes) & 684 & 81.4 & 495 & 81.1 & 189 & 82.2 & 0.733 \\
\hline
\end{tabular}

Do you think that the measures put in place

by the health authorities to contain the

spread of the pandemic in Italy are 
Table 2. Cont.

\begin{tabular}{|c|c|c|c|c|c|c|c|}
\hline & \multicolumn{2}{|c|}{$\begin{array}{c}\text { Total } \\
(n=958)\end{array}$} & \multicolumn{2}{|c|}{$\begin{array}{l}\text { Pre-Lockdown } \\
\quad(n=694)\end{array}$} & \multicolumn{2}{|c|}{$\begin{array}{l}\text { Lockdown } \\
(n=264)\end{array}$} & \multirow[b]{2}{*}{$p$} \\
\hline & $\mathbf{n}$ & $\%$ & $\mathbf{n}$ & $\%$ & $\mathbf{n}$ & $\%$ & \\
\hline Yes, absolutely & 211 & 25.0 & 149 & 24.3 & 62 & 27.1 & \\
\hline Yes, moderately & 395 & 46.9 & 284 & 46.2 & 111 & 48.5 & \\
\hline No, not really & 181 & 21.5 & 141 & 23.0 & 40 & 17.5 & \\
\hline No, not at all & 44 & 5.2 & 30 & 4.9 & 14 & 6.1 & \\
\hline I don't know & 12 & 1.4 & 10 & 1.6 & 2 & 0.9 & \\
\hline $\begin{array}{l}\text { Now that the COVID-19 pandemic has } \\
\text { arrived in Italy, do you feel ready to face it? }\end{array}$ & & & & & & & 0.234 \\
\hline Yes, absolutely & 61 & 7.3 & 40 & 6.6 & 21 & 9.3 & \\
\hline Yes, moderately & 308 & 37.0 & 216 & 35.6 & 92 & 40.7 & \\
\hline No, not really & 351 & 42.1 & 266 & 43.8 & 85 & 37.6 & \\
\hline No, not at all & 94 & 11.3 & 69 & 11.4 & 25 & 11.1 & \\
\hline I don't know & 19 & 2.3 & 16 & 2.6 & 3 & 1.3 & \\
\hline
\end{tabular}

With reference to the COVID-19 pandemic,

do you think that the importance given to, and spread by, the media and society in general is excessive?

\begin{tabular}{cccccccc}
\hline Yes, absolutely & 131 & 16.0 & 107 & 18.0 & 24 & 10.6 \\
\hline Yes, moderately & 216 & 26.3 & 167 & 28.1 & 49 & 21.7 \\
\hline No, not really & 265 & 32.3 & 186 & 31.3 & 79 & 35.0 & 32.3 \\
\hline No, not at all & 199 & 24.3 & 126 & 21.2 & 73 & 1 & 0.4 \\
\hline I don't know & 9 & 1.1 & 8 & 1.4 & 1.4 \\
\hline
\end{tabular}

${ }^{*}$ more than one answer was possible.

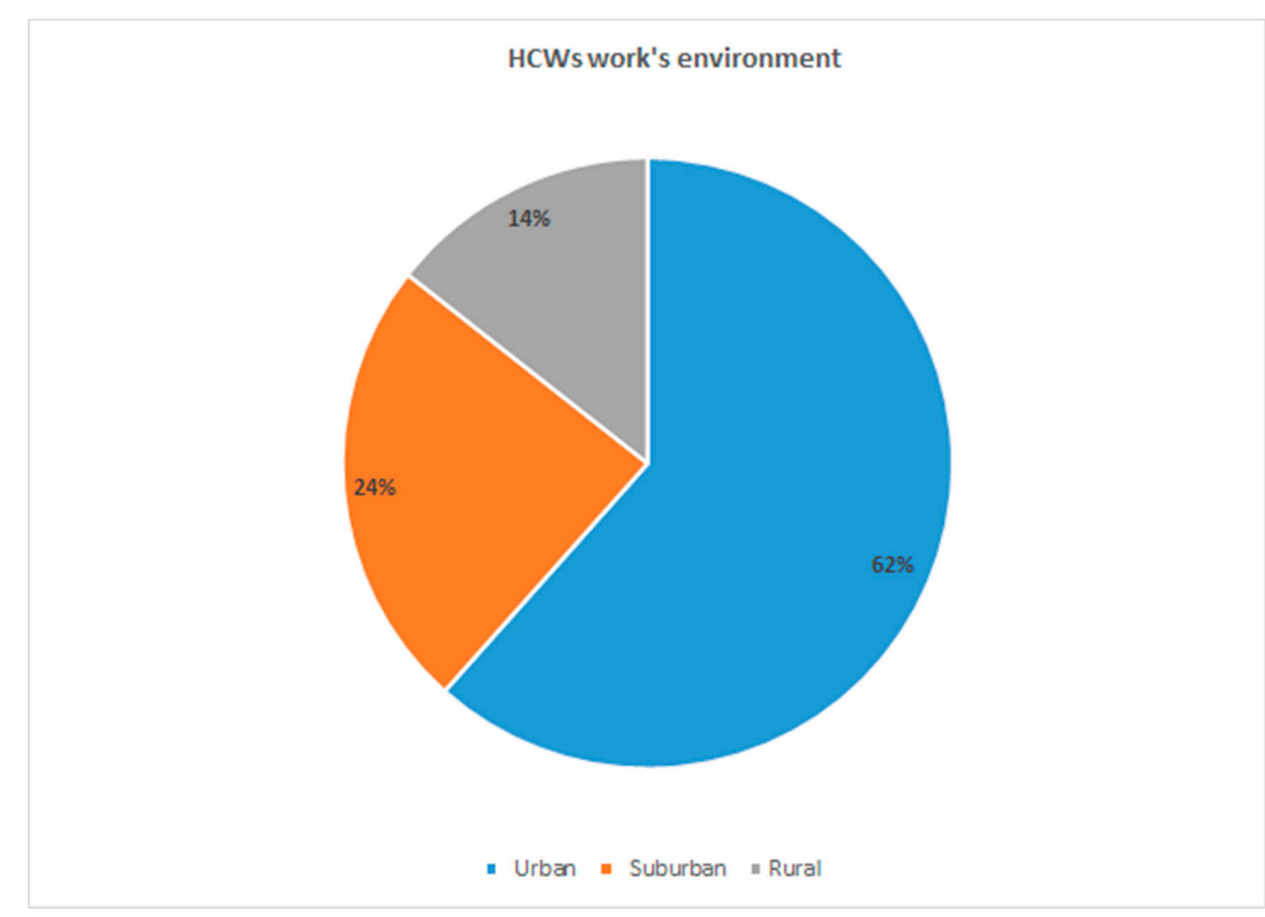

Figure 1. Healthcare workers' environment of work. 


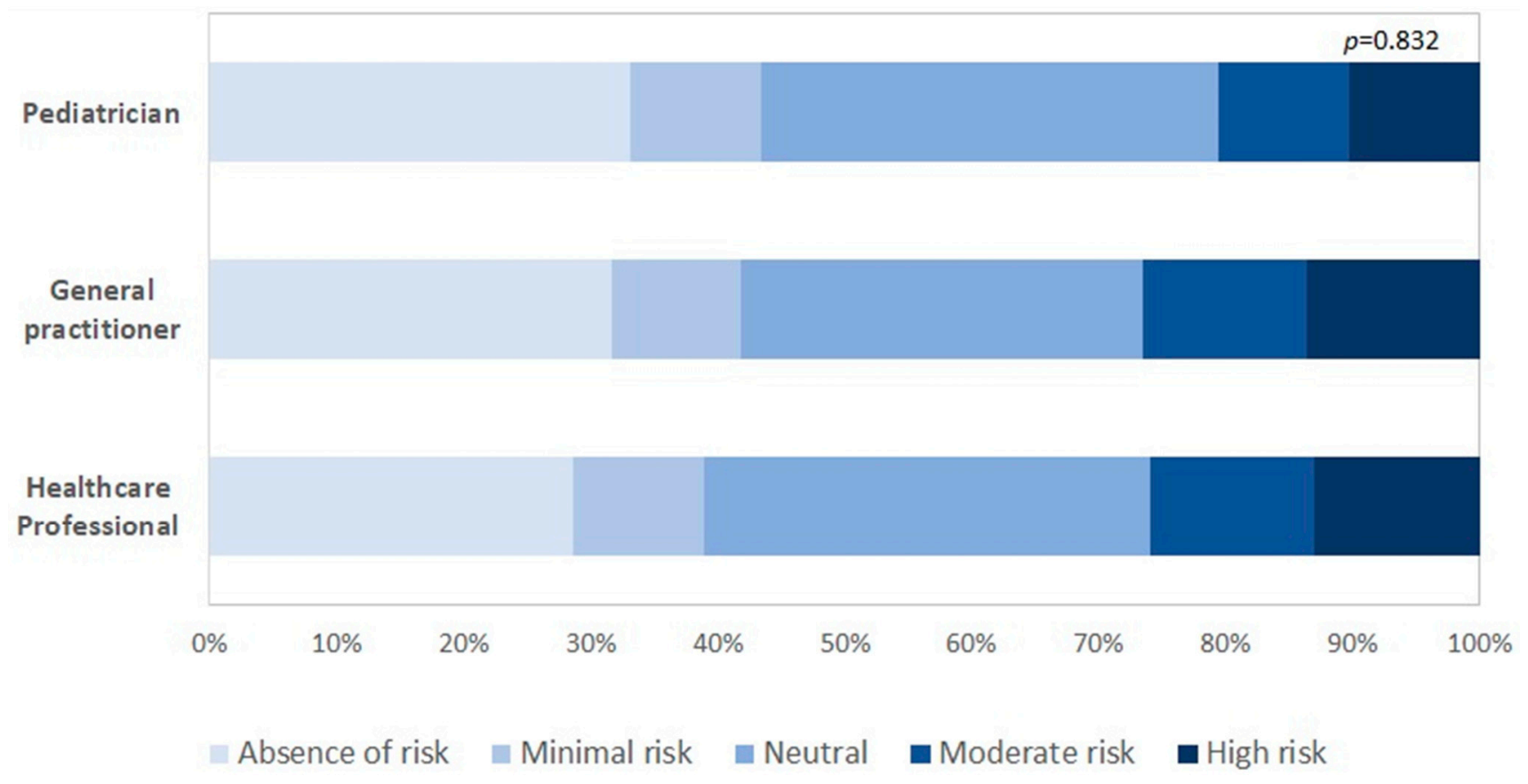

Figure 2. Proportion of HCWs reporting patients afraid of the novel coronavirus pandemic.

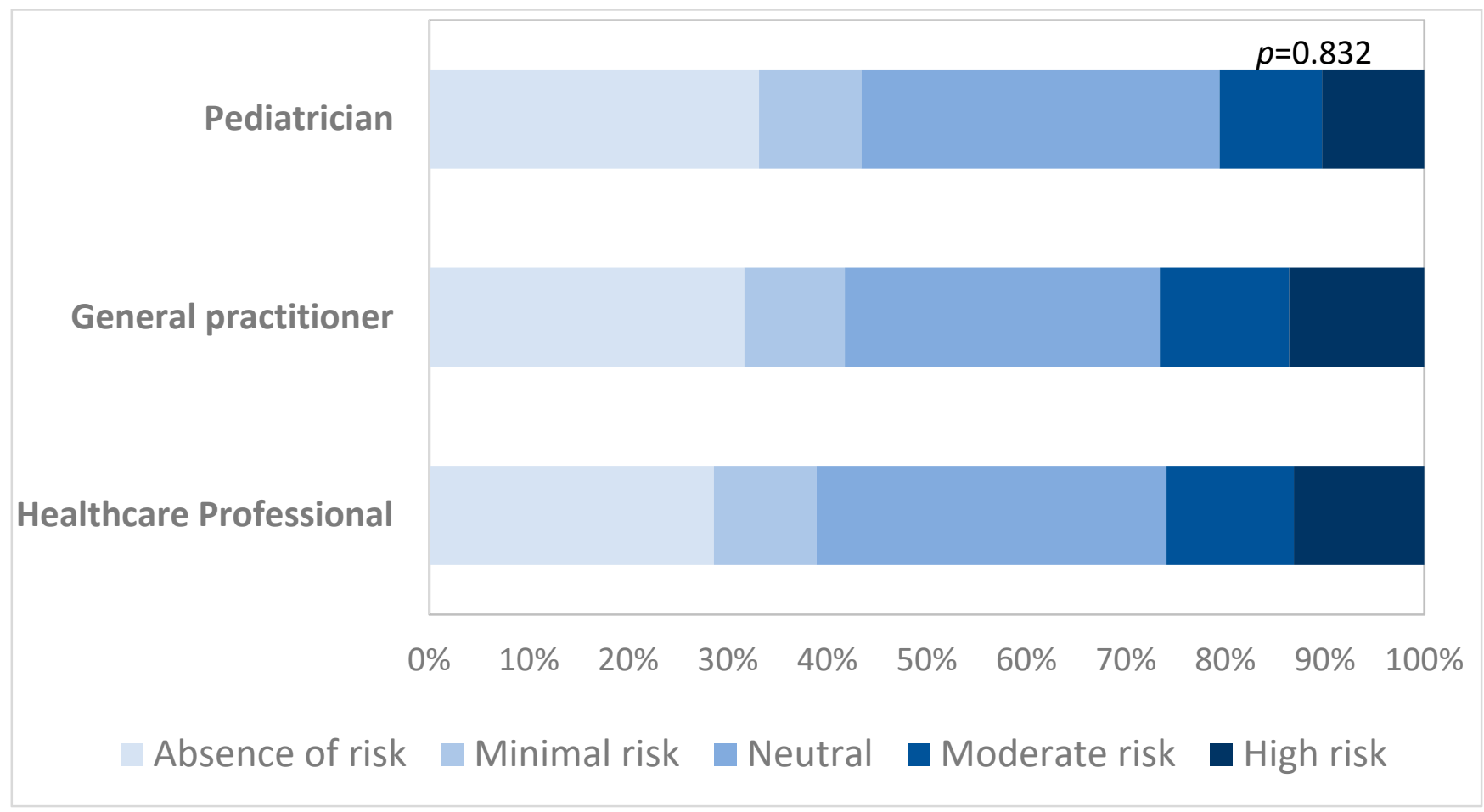

Figure 3. Perception of the risk to visit a COVID-19 patient by type of HCWs.

Respondents reported having changed their clinical practice more in the lockdown period $(81.1 \%)$ than in pre-lockdown (46.3\%) (Table 2). Particularly, they increased the use of masks $(87.1 \%, p<0.001)$, disinfection and sanitization of doctors' offices $(75.8 \%$, $p<0.001)$, the use of protective glasses and alcoholic hand solution $(71.2 \%, p<0.001)$ and hand washing $(31.8 \%, p=0.028)$ (Table 2$)$.

Among participants, pediatricians were those who felt most well informed by health authorities $(96.4 \%)$. 
The multivariate model analyzing information showed that, in the older age groups, information increases with age, particularly in respondents aged over 66 (OR 2.03, $p=0.040$ ). Pediatricians are the most well informed (OR 1.78, $p=0.015)$ and institutional e-mails are the most reported method for them to be informed (OR 1.81, $p<0.001$ ). Participants who declared not to feel ready to face the COVID-19 emergency also reported less information (OR 0.13, $p<0.001$ ) (Table 3).

The multivariate model analyzing behavior change showed that general practitioners changed their behavior less than health professionals (OR 0.54, $p=0.008$ ). Participants from Southern Italy changed their behavior less than those from Northern Italy (OR 0.67, $p=0.049$ ). Age positively affected behavior change, and respondents ranging from 46 to 55 years $(\mathrm{OR}=1.81, p=0.024)$ of age showed more willingness to change their behavior, as did those aged 56-65 (OR $=1.81, p=0.023)$ (Table 3).

Respondents declared to have changed their behavior more during the lockdown period than pre-lockdown (OR 6.22, $p<0.001)$. Moreover, those who used the ministry toll-free number to inform themselves, reported the greatest behavior change (OR 2.03, $p=0.001$ ).

Table 3. Odd ratios of information and behavioral change.

\begin{tabular}{|c|c|c|c|c|c|c|}
\hline & \multicolumn{3}{|c|}{ Information } & \multicolumn{3}{|c|}{ Behavior Change } \\
\hline & adjOR & $p$-Value & $95 \% \mathrm{CI}$ & $\operatorname{adjOR}$ & $p$-Value & $95 \% \mathrm{CI}$ \\
\hline \multicolumn{7}{|l|}{ Age } \\
\hline$\leq 35$ & Reference & & & Reference & & \\
\hline $36-45$ & 0.89 & 0.614 & $0.56-1.42$ & 1.71 & $0.027 *$ & $1.06-2.76$ \\
\hline $46-55$ & 1.10 & 0.701 & $0.67-1.80$ & 1.81 & $0.024 *$ & $1.08-3.04$ \\
\hline $56-65$ & 1.60 & 0.061 & $0.98-2.62$ & 1.81 & $0.023 *$ & $1.09-3.02$ \\
\hline$\geq 66$ & 2.03 & $0.040 *$ & $1.03-4.00$ & 1.49 & 0.241 & $0.76-2.93$ \\
\hline \multicolumn{7}{|l|}{ Sex } \\
\hline Male & Reference & & & Reference & & \\
\hline Female & 1.23 & 0.225 & $0.88-1.70$ & 1.26 & 0.184 & $0.90-1.78$ \\
\hline \multicolumn{7}{|l|}{ Profession } \\
\hline Healthcare Professional & Reference & & & Reference & & \\
\hline General practitioner & 1.03 & 0.886 & $0.67-1.58$ & 0.54 & $0.008 *$ & $0.35-0.85$ \\
\hline Pediatrician & 1.78 & $0.015 *$ & $1.12-2.85$ & 0.64 & 0.083 & $0.39-1.07$ \\
\hline \multicolumn{7}{|l|}{ Region } \\
\hline North Italy & Reference & & & Reference & & \\
\hline Central Italy & 1.35 & 0.078 & $0.97-1.88$ & 0.73 & 0.066 & $0.51-1.02$ \\
\hline South Italy & 1.41 & 0.076 & $0.93-2.08$ & 0.67 & 0.049 * & $0.46-1.00$ \\
\hline \multicolumn{7}{|l|}{ Period } \\
\hline Pre-lockdown & Reference & & & Reference & & \\
\hline Lockdown & 1.16 & 0.377 & $0.84-1.59$ & 6.22 & $<0.001 *$ & $4.22-9.17$ \\
\hline \multicolumn{7}{|l|}{$\begin{array}{c}\text { Email sent by health } \\
\text { authorities }\end{array}$} \\
\hline No & Reference & & & Reference & & \\
\hline Yes & 1.81 & $<0.001 *$ & $1.32-2.50$ & 1.28 & 0.153 & $0.91-1.79$ \\
\hline \multicolumn{7}{|l|}{$\begin{array}{l}\text { Ministry of Health } \\
\text { toll-free number }\end{array}$} \\
\hline No & Reference & & & Reference & & \\
\hline Yes & 1.21 & 0.304 & $0.84-1.73$ & 2.03 & 0.001 * & $1.35-3.06$ \\
\hline \multicolumn{7}{|l|}{$\begin{array}{l}\text { Now that the pandemic } \\
\text { from COVID-19 has } \\
\text { arrived in Italy, do you } \\
\text { feel ready to face it? }\end{array}$} \\
\hline Yes, absolutely & Reference & & & Reference & & \\
\hline Yes, moderately & 0.56 & 0.064 & $0.31-1.03$ & 1.88 & 0.028 * & $1.07-3.30$ \\
\hline No, not really & 0.27 & $<0.001$ * & $0.15-0.50$ & 1.76 & $0.047^{*}$ & $1.01-3.08$ \\
\hline No, not at all & 0.13 & $<0.001$ * & $0.06-0.26$ & 1.35 & 0.390 & $0.68-2.64$ \\
\hline I don't know & 0.20 & $0.003 *$ & $0.07-0.57$ & 1.82 & 0.288 & $0.60-5.51$ \\
\hline
\end{tabular}

* significant $p$-value. The model was adjusted also for sex, email sent by health authorities, official institutions' websites, emails received from scientific companies, exchange of information with other colleagues, medical-scientific publications, social network. 


\section{Discussion}

COVID-19 is a global health problem, especially among HCWs. Italy was the first European country to face COVID-19 pandemic, with considerable differences in terms of organization and management strategies throughout the country, resulting in heterogeneous levels of performance across regional health systems.

Our study shows that HCWs have a sufficient level of information about COVID-19, and participants frequently reported a change in their behavior in clinical practice during the pandemic. Doctors had a higher level of information and, amongst those, pediatricians were better informed.

Healthcare professionals, being in contact with patients, played a crucial role in the transmission of COVID-19; thousands of HCW's, mainly general practitioners, were affected by COVID-19 and died while caring for COVID-19 positive patients [26]. This could be due to several factors: lack of personal protective equipment (PPE), poor information of the virus containment measures, especially in the first pandemic wave, and heavy workloads. For this reason, we investigated HCWs' level of information and perceptions of the prevention and control of the COVID-19 pandemic. It is crucial for HCWs to be prepared and to apply all IPC in facing COVID-19 [29-31] considering that the prevalence of the infection among HCWs exceeded 10\% in Italy [32-34] with a consequent loss of capacity for hospitals to respond adequately to the pandemic.

Information and perceptions of COVID-19 varied across different categories of HCWs. Other studies have shown that the majority of HCWs had a good level of information on COVID-19 and showed a positive attitude related to their sense of readiness to confront the disease and implemented good practices towards COVID-19 [30,35-37].

Moreover, doctors declared they had significantly modified their clinical practice during the pandemic period compared to other health professionals, showing a higher impact of the pandemic on medical doctors' daily routines. HCWs needed deeper knowledge, and they tried to obtain this knowledge through national health authorities, other colleagues' opinions or social networks.

Doctors were also much more confident in the information coming from the Italian National Health Authorities compared to other health professionals. This indicates that the COVID-19-related updates posted by official health authorities had positive implications for improving doctors' information levels. Obtaining information from institutional sources is crucial for disseminating reliable data about the emerging COVID-19 infection and is essential for HCWs' preparedness and response [29].

During the pre-lockdown and lockdown periods, all health professionals were informed about the best IPC to be adopted for the containment of COVID-19. Most respondents received their information from institutional channels, while $38.5 \%$ had obtained information from other colleagues and $15.8 \%$ from social networks.

The findings of this study suggest a significant gap between the amount of information available on COVID-19 and the depth of information among HCWs, particularly regarding disinfection of doctors' offices and contact surfaces, use of protective glasses and use of alcoholic solution for hand hygiene. All these practices were not extensively applied in the pre-lockdown period because they were not routinely used by HCWs before the pandemic, yet their use significantly increased during lockdown. This could be due to a low penetration of information and trust in the messages of the health authorities at the beginning of the pandemic, which were perceived only later as important and vital to fight the pandemic. However, it is important to note that, as expected, the change in IPC measures was mainly driven by those measures less used in the clinical routine (such as use of protective glasses, and use of alcoholic solution for hand hygiene), while well known IPC measures (such as hand washing) increased less.

Other studies, investigating scope of information level, risk perception and practices, showed that doctors exhibited higher knowledge scores than nurses and paramedics [30]. Information, risk perception and practice regarding the use of masks and their differences were found to be inadequate mostly among medical staff. 
HCWs showed a positive attitude but moderate-to-poor level of information and practice regarding the use of face masks $[35,38,39]$.

Two interesting results in our study were that the majority of respondents declared to have radically changed their work habits in the lockdown period; moreover, the use of surgical masks among respondents increased much more in the lockdown period compared to the use of FFP1 or FFP3 masks.

However, this study has some limitations that should be considered. The survey was conducted through the use of an online platform and disseminated through social networks; therefore, the type of sampling used may not be representative of all Italian HCWs and could be biased towards respondents with a positive knowledge, attitude and practice.

\section{Conclusions}

We identified a good level of information among respondent HCWs who felt to be adequately prepared to deal with the pandemic. However, considering that the global threat of COVID-19 continues to emerge and that healthcare professionals are at high risk of COVID-19 transmission to and from patients, greater efforts through educational campaigns that target HCWs are urgently needed.

Author Contributions: C.R., I.C. (Ilaria Campagna) and E.P. contributed to the study design and to the first draft of the manuscript; I.C. (Ileana Croci) carried out statistical analysis and contributed in writing the methods section; L.R. (Luisa Russo), S.C., F.G. and A.E.T. contributed in drafting and reviewing the manuscript; L.R. (Lara Ricotta), M.R., and M.L.C.D.A. contributed to the study conceptualization and reviewed the final draft of the manuscript. All authors have read and agreed to the published version of the manuscript.

Funding: This research received no external funding.

Institutional Review Board Statement: The study was conducted according to the guidelines of the Declaration of Helsinki, and approved by the Institutional Review Board (or Ethics Committee) of Bambino Gesù Children Hospital 's Ethical Committee (Prot n. 2116/2020).

Informed Consent Statement: Informed consent was obtained from all subjects involved in the study before the participants were able to access the online survey questionnaire. Confidentiality and anonymity were maintained through the settings of the online survey.

Data Availability Statement: All data generated or analyzed during this study are included in this published.

Acknowledgments: We thank all healthcare workers who, even in a very difficult professional and personal moment, participated in the survey, thus making the study possible.

Conflicts of Interest: The authors declare that they have no conflict of interest.

\begin{tabular}{ll}
\multicolumn{2}{l}{ Abbreviations } \\
HCW & Healthcare workers \\
IPC & Infection prevention and control \\
COVID-19 & Coronavirus Disease 2019 \\
PPE & Personal protective equipment
\end{tabular}

\section{References}

1. Zhu, N.; Zhang, D.; Wang, W.; Li, X.; Yang, B.; Song, J.; Zhao, X.; Huang, B.; Shi, W.; Lu, R.; et al. A Novel Coronavirus from Patients with Pneumonia in China, 2019. N. Engl. J. Med. 2020, 382, 727-733. [CrossRef]

2. WHO. Director-General's opening remarks at the media briefing on COVID-19-11 March 2020. Available online: https: / / www.who.int/director-general/speeches / detail/who-director-general-s-opening-remarks-at-the-media-briefing-on-covid19---11-march-2020 (accessed on 16 November 2020).

3. Lescure, F.-X.; Bouadma, L.; Nguyen, D.; Parisey, M.; Wicky, P.-H.; Behillil, S.; Gaymard, A.; Bouscambert-Duchamp, M.; Donati, F.; Le Hingrat, Q.; et al. Clinical and virological data of the first cases of COVID-19 in Europe: A case series. Lancet Infect. Dis. 2020, 20, 697-706. [CrossRef] 
4. Spiteri, G.; Fielding, J.; Diercke, M.; Campese, C.; Enouf, V.; Gaymard, A.; Bella, A.; Sognamiglio, P.; Moros, M.J.S.; Riutort, A.N.; et al. First cases of coronavirus disease 2019 (COVID-19) in the WHO European Region, 24 January to 21 February 2020. Euro Surveill. 2020, 25, 2000178. Available online: https://www.ncbi.nlm.nih.gov/pmc/articles/PMC7068164/ (accessed on 16 November 2020). [CrossRef]

5. Giovanetti, M.; Benvenuto, D.; Angeletti, S.; Ciccozzi, M. The first two cases of 2019-nCoV in Italy: Where they come from? J. Med. Virol. 2020, 92, 518-521. Available online: https://www.ncbi.nlm.nih.gov/pmc/articles/PMC7166327/ (accessed on 16 November 2020). [CrossRef] [PubMed]

6. Guzzetta, G.; Poletti, P.; Ajelli, M.; Trentini, F.; Marziano, V.; Cereda, D.; Tirani, M.; Diurno, G.; Bodina, A.; Barone, A.; et al. Potential short-term outcome of an uncontrolled COVID-19 epidemic in Lombardy, Italy, February to March 2020. Eurosurveillance 2020, 25, 2000293. Available online: https://www.eurosurveillance.org/content/10.2807/1560-7917.ES.2020.25.12.2000293 (accessed on 16 November 2020). [CrossRef] [PubMed]

7. Indolfi, C.; Spaccarotella, C. The Outbreak of COVID-19 in Italy. JACC Case Rep. 2020, 2, 1414-1418. [CrossRef] [PubMed]

8. Backer, J.A.; Klinkenberg, D.; Wallinga, J. Incubation period of 2019 novel coronavirus (2019-nCoV) infections among travellers from Wuhan, China, 20-28 January 2020. Euro Surveill. 2020, 25, 2000062. [CrossRef] [PubMed]

9. Decreto Del Presidente Del Consiglio Dei Ministri. Available online: https://www.trovanorme.salute.gov.it/norme/ dettaglioAtto?id=73196\&articolo=6 (accessed on 23 February 2020).

10. Decreto Del Presidente Del Consiglio Dei Ministri. Available online: https://www.trovanorme.salute.gov.it/norme/ dettaglioAtto?id=73643 (accessed on 11 March 2020).

11. Lai, A.; Bergna, A.; Caucci, S.; Clementi, N.; Vicenti, I.; Dragoni, F.; Cattelan, A.M.; Menzo, S.; Pan, A.; Callegaro, A.; et al. Molecular tracing of SARS-CoV-2 in Italy in the first three months of the epidemic. Viruses 2020, 12, 798. Available online: https: / / covid19.elsevierpure.com/it/publications/molecular-tracing-of-sars-cov-2-in-italy-in-the-first-three-month (accessed on 16 November 2020). [CrossRef]

12. Onder, G.; Giovanni, R.; Silvio, B. Case-Fatality Rate and Characteristics of Patients Dying in Relation to COVID-19 in Italy. JAMA 2020, 323, 1775-1776. Available online: http:/ / europepmc.org/article/MED/32203977 (accessed on 16 November 2020). [CrossRef] [PubMed]

13. Gámbaro, F.; Behillil, S.; Baidaliuk, A.; Donati, F.; Albert, M.; Alexandru, A.; Vanpeene, M.; Bizard, M.; Brisebarre, A.; Barbet, M.; et al. Introductions and early spread of SARS-CoV-2 in France, 24 January to 23 March 2020. Eurosurveillance 2020, 25, 2001200. Available online: https:/ / www.eurosurveillance.org/content/10.2807/1560-7917.ES.2020.25.26.2001200 (accessed on 16 November 2020). [CrossRef]

14. Guan, W.J.; Ni, Z.Y.; Hu, Y.; Liang, W.H.; Ou, C.Q.; He, J.X.; Liu, L.; Shan, H.; Lei, C.L.; Hui, D.S.C.; et al. Clinical Characteristics of Coronavirus Disease 2019 in China. N. Engl. J. Med. 2020. [CrossRef]

15. Shah, S.J.; Barish, P.N.; Prasad, P.A.; Kistler, A.; Neff, N.; Kamm, J.; Li, L.M.; Chiu, C.Y.; Babik, J.M.; Fang, M.C.; et al. Clinical features, diagnostics, and outcomes of patients presenting with acute respiratory illness: A retrospective cohort study of patients with and without COVID-19. EclinicalMedicine 2020, 27, 100518. Available online: https:/ /www.ncbi.nlm.nih.gov/pmc/articles/ PMC7447618/ (accessed on 16 November 2020). [CrossRef]

16. Wang, D.; Hu, B.; Hu, C.; Zhu, F.; Liu, X.; Zhang, J.; Wang, B.; Xiang, H.; Cheng, Z.; Xiong, Y.; et al. Clinical Characteristics of 138 Hospitalized Patients With 2019 Novel Coronavirus-Infected Pneumonia in Wuhan, China. JAMA 2020, 323, 1061-1069. [CrossRef]

17. Infection Prevention and Control During Health Care when Coronavirus Disease (COVID-19) is Suspected or Confirmed. Available online: https:/ / www.who.int/publications-detail-redirect/WHO-2019-nCoV-IPC-2020.4 (accessed on 16 November 2020).

18. Lan, F.-Y.; Filler, R.; Mathew, S.; Buley, J.; Iliaki, E.; Bruno-Murtha, L.A.; Osgood, R.; Christophi, C.A.; Fernandez-Montero, A.; Kales, S.N. COVID-19 symptoms predictive of healthcare workers' SARS-CoV-2 PCR results. PLoS ONE 2020, 15. Available online: https: / / www.ncbi.nlm.nih.gov/pmc/articles/PMC7319316/ (accessed on 17 November 2020). [CrossRef]

19. Fusco, F.; Pisaturo, M.; Iodice, V.; Bellopede, R.; Tambaro, O.; Parrella, G.; Di Flumeri, G.; Viglietti, R.; Pisapia, R.; Carleo, M.; et al. COVID-19 among healthcare workers in a specialist infectious diseases setting in Naples, Southern Italy: Results of a crosssectional surveillance study. J. Hosp. Infect. 2020, 105, 596-600. Available online: https://www.ncbi.nlm.nih.gov/pmc/articles/ PMC7301109/ (accessed on 17 November 2020). [CrossRef]

20. El-Boghdadly, K.; Wong, D.J.N.; Owen, R.; Neuman, M.D.; Pocock, S.; Carlisle, J.B.; Johnstone, C.; Andruszkiewicz, P.; Baker, P.A.; Biccard, B.M.; et al. Risks to healthcare workers following tracheal intubation of patients with COVID-19: A prospective international multicentre cohort study. Anaesthesia 2020, 75, 1437-1447. [CrossRef]

21. Chen, Y.; Tong, X.; Wang, J.; Huang, W.; Yin, S.; Huang, R.; Yang, H.; Chen, Y.; Huang, A.; Liu, Y.; et al. High SARS-CoV-2 antibody prevalence among healthcare workers exposed to COVID-19 patients. J. Infect. 2020, 81, 420-426. Available online: https: / / www.ncbi.nlm.nih.gov/pmc/articles/PMC7270786/ (accessed on 17 November 2020). [CrossRef]

22. Wu, Z.; McGoogan, J.M. Characteristics of and Important Lessons From the Coronavirus Disease 2019 (COVID-19) Outbreak in China: Summary of a Report of 72-314 Cases From the Chinese Center for Disease Control and Prevention. JAMA Netw. 2020, 323, 1239-1242. [CrossRef] [PubMed]

23. Report of the WHO-China Joint Mission on Coronavirus Disease 2019 (COVID-19). Available online: https://www.who. int/ publications-detail-redirect/report-of-the-who-china-joint-mission-on-coronavirus-disease-2019-(covid-19) (accessed on 16 November 2020). 
24. Chou, R.; Dana, T.; Buckley, D.I.; Selph, S.; Fu, R.; Totten, A.M. Epidemiology of and Risk Factors for Coronavirus Infection in Health Care Workers: A Living Rapid Review. Ann. Intern. Med. 2020, 173, 120-136. [CrossRef] [PubMed]

25. Rapporto Istituto Superiore di Sanità COVID-19 n. 2/2020 Rev. 2.21. Available online: https://www.iss.it/rapporti-covid-19//asset_publisher/btw1J82wtYzH/content/indicazioni-ad-interim-per-un-utilizzo-razionale-delle-protezioni-per-infezioneda-sars-cov-2-nelle-attivit\%25C3\%25A0-sanitarie-e-sociosanitarie-assistenza-a-soggetti-affetti-da-covid-19-nell-attualescenario-emergenziale-sars-cov-2 (accessed on 16 November 2020).

26. DECRETO-LEGGE 9 marzo 2020, n. 14. Disposizioni urgenti per il potenziamento del Servizio sanitario nazionale in relazione all'emergenza COVID-19. Available online: https://www.gazzettaufficiale.it/eli/gu/2020/03/09/62/sg/pdf (accessed on 17 November 2020).

27. White, I.R.; Royston, P.; Wood, A.M. Multiple imputation using chained equations: Issues and guidance for practice. Stat. Med. 2011, 30, 377-399. [CrossRef] [PubMed]

28. Moons, K.G.M.; Donders, R.A.R.T.; Stijnen, T.; Harrell, F.E. Using the outcome for imputation of missing predictor values was preferred. J. Clin. Epidemiol. 2006, 59, 1092-1101. [CrossRef] [PubMed]

29. Bhagavathula, A.S.; Aldhaleei, W.A.; Rahmani, J.; Mahabadi, M.A.; Bandari, D.K. Knowledge and Perceptions of COVID-19 Among Health Care Workers: Cross-Sectional Study. JMIR Public Health Surveill. 2020, 6, e19160. [CrossRef] [PubMed]

30. Zhang, M.; Zhou, M.; Tang, F.; Wang, Y.; Nie, H.; Zhang, L.; You, G. Knowledge, attitude, and practice regarding COVID-19 among healthcare workers in Henan, China. J. Hosp. Infect. 2020, 105, 183-187. [CrossRef] [PubMed]

31. Sommerstein, R.; Fux, C.A.; Vuichard-Gysin, D.; Abbas, M.; Marschall, J.; Balmelli, C.; Troillet, N.; Harbarth, S.; Schlegel, M.; Schlegel, M.; et al. Risk of SARS-CoV-2 transmission by aerosols, the rational use of masks, and protection of healthcare workers from COVID-19. Antimicrob. Resist. Infect. Control. 2020, 9, 100. [CrossRef]

32. Istituto Superiore di Sanità- Sorveglianza Integrata COVID-19 in Italia (Ordinanza n. 640 del 27/02/2020). Available online: https:/ / www.epicentro.iss.it/coronavirus/bollettino/Infografica_7aprile\%20ITA.pdf (accessed on 17 November 2020).

33. Boccia, S.; Ricciardi, W.; Ioannidis, J.P.A. What Other Countries Can Learn From Italy During the COVID-19 Pandemic. JAMA Intern. Med. 2020, 180, 927-928. [CrossRef]

34. Colaneri, M.; Novelli, V.; Cutti, S.; Muzzi, A.; Resani, G.; Monti, M.C.; Rona, C.; Grugnetti, A.M.; Rettani, M.; Rovida, F.; et al The experience of the health care workers of a severely hit SARS-CoV-2 referral Hospital in Italy: Incidence, clinical course and modifiable risk factors for COVID-19 infection. J Public Health 2020. [CrossRef]

35. Saqlain, M.; Munir, M.; Rehman, S.; Gulzar, A.; Naz, S.; Ahmed, Z.; Tahir, A.; Mashhood, M. Knowledge, attitude, practice and perceived barriers among healthcare workers regarding COVID-19: A cross-sectional survey from Pakistan. J. Hosp. Infect. 2020, 105, 419-423. Available online: https:/ / www.ncbi.nlm.nih.gov/pmc/articles/PMC7211584/ (accessed on 17 November 2020).

36. Huynh, G.; Nguyen, T.; Tran, V.; Vo, K.; Vo, V.; Pham, L. Knowledge and attitude toward COVID-19 among healthcare workers at District 2 Hospital, Ho Chi Minh City. Asian Pac. J. Trop. Med. 2020, 260-265. [CrossRef]

37. Salman, M.; Mustafa, Z.; Asif, N.; Zaidi, H.A.; Shehzadi, N.; Khan, T.M.; Saleem, Z.; Hussain, K. Knowledge, attitude and preventive practices related to COVID-19 among health professionals of Punjab province of Pakistan. J. Infect. Dev. Ctries. 2020, 14, 707-712. [CrossRef]

38. Kumar, J.; Katto, M.S.; A Siddiqui, A.; Sahito, B.; Jamil, M.; Rasheed, N.; Ali, M. Knowledge, Attitude, and Practices of Healthcare Workers Regarding the Use of Face Mask to Limit the Spread of the New Coronavirus Disease (COVID-19). Cureus 2020, 12 , e7737. [CrossRef]

39. Piché-Renaud, P.-P.; Groves, H.E.; Kitano, T.; Arnold, C.; Thomas, A.; Streitenberger, L.; Alexander, L.; Morris, S.K. Healthcare worker perception of a global outbreak of novel coronavirus (COVID-19) and personal protective equipment: Survey of a pediatric tertiary-care hospital. Infect. Control. Hosp. Epidemiol. 2020, 1-7. [CrossRef] 